\title{
A Versatile Ratio Instrument for the High Ratio Comparison of Voltage or Resistance
}

\author{
Alfred E. Hess* \\ Institute for Basic Standards, National Bureau of Standards, Boulder, Colo.
}

(April 12, 1966)

\begin{abstract}
A 9-dial resistance ratio instrument capable of precise high ratio comparison of voltage or resistance (from $1 / 1$ to $10^{7} / 1$ ) is described. Also described is the modification of a 6-dial universal ratio set to permit its additional use as a versatile ratio instrument. Paramount to the accuracy of these high ratio instruments is the carefully adjusted "common point" junction which is briefly discussed.
\end{abstract}

Key Words: DRRS (direct reading ratio set); junction; precision measurements; ratio, resistor, four-terminal; resistance decade; transresistance; URS (universal ratio set); VERI (versatile ratio instrument).

\section{Introduction}

Because of a need to make accurate high ratio d-c resistance comparisons and an inability to do so with existing ratio instruments such as the DRRS (direct reading ratio set) and the URS (universal ratio set), $[1,2,3]^{1}$ a versatile ratio instrument (abbreviated VERI) was developed. With the VERI an arbitrary voltage or resistance ratio from $1 / 1$ to $10^{7} / 1$ (or $1 / 10^{7}$ ) may be measured with a dial resolution of 1 part in $10^{8}$ or better. (Permissible generator voltage and detector sensitivity may further limit the actual resolution attainable.) This instrument may be used to calibrate ratio devices-potentiometers, voltage dividers, volt boxes, the ratio arms of Wheatstone bridges, etc. Also, the individual fixed resistors of the VERI may be used as 4-terminal resistance standards, and the incremental resistance section may be used as a 9dial, 2- or 4-terminal resistance decade.

A 6-dial URS may be modified as described in section 3 to permit its additional use as a 6-dial VERI having a ratio range from $1 / 1$ to $2 \times 10^{4} / 1$. Its normal function as a URS is not impaired by the modification.

The accuracy of the VERI depends directly upon the design and adjustment of the "common point" junction [4]. The transresistances ${ }^{2}$ of the junctions used in the described instruments are less than $10^{-8} \Omega$. This causes a worst-case uncertainty in ratio of less than 1 part in $10 .^{7}$

\footnotetext{
*Radio Standards Engineering Division, National Bureau of Standards, Boulder, Colo.

${ }^{1}$ Figures in brackets indicate the literature references at the end of this paper.

${ }^{2}$ Transresistance: The ratio of the voltage induced at one pair of junction terminals to the current through another terminal pair, i.e., $R_{1243}=\frac{E_{24}}{I_{13}}$, see figure 1 .
}

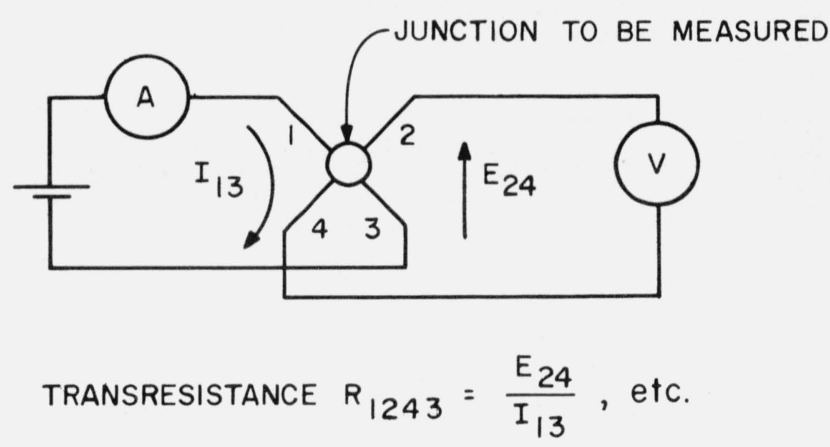

FIGURE 1. Basic setup for measuring junction transresistance.

\section{Versatile Ratio Instrument}

Figure 2 is a schematic diagram of the VERI. It consists basically of a precision resistance decade section and several precision fixed resistors of decimal values connected at a common point by a "zero" transresistance junction. The resistance decade section shown consists of 9 decades which may be varied in $0.001 \Omega$ steps to a total resistance of $1,111,111.111$ $\Omega$. The seven fixed resistors have decimal values from 0.1 to $100 \mathrm{k} \Omega$. The "outside" ends of each of the resistors have dual terminals, so they may be connected as 4-terminal resistors to permit the elimination of the terminal and external lead resistances. The "common-point" junction shown is a 


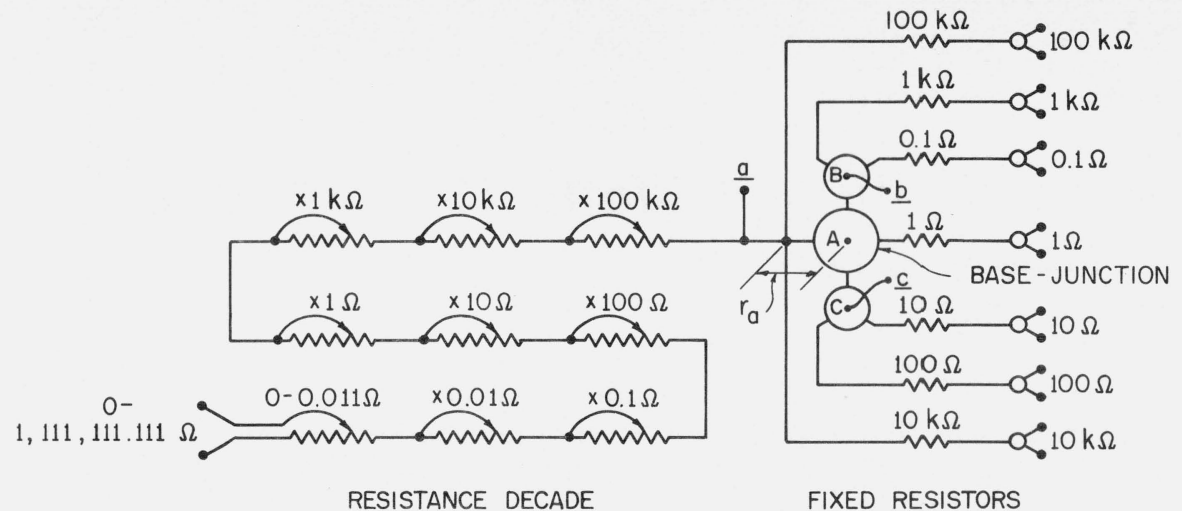

FIGURE 2. Schematic diagram of a versatile resistance ratio instrument.

composite junction consisting of three tetrajunctions, ${ }^{3}$ $\mathrm{A}, \mathrm{B}$, and $\mathrm{C}$. This is necessary because a junction may have a maximum of only four electrically symmetrical terminals [4]. Junction $A$ is the base junction to which all resistance and ratio measurements are referred. It is adjusted to have less than $10^{-8} \Omega$ transresistance. Because of their relatively large resistance, the $10 \mathrm{k} \Omega$ and $100 \mathrm{k} \Omega$ fixed resistors, and the $0-1 \mathrm{M} \Omega$ resistance decade section may be connected to a common terminal of the base juction without introducing significant error (assuming $r_{a}$ is less than $0.001 \Omega$ ). Terminals $\mathrm{a}, \mathrm{b}$, and $\mathrm{c}$ are needed for the calibration and use of the VERI since the potential terminal used for a ratio measurement should not be common to either of the resistors being used in the VERI. The switches used in the resistance decades have low resistance variation (less than $0.001 \Omega$ per switch).

This instrument when fully calibrated may be used in a variety of ways, including:

(1) 4-terminal fixed resistance standards, 0.1, 1, $10,100,1 \mathrm{k}, 10 \mathrm{k}$, and $100 \mathrm{k} \Omega$;

(2) a 2- or 4-terminal, 9-dial decade resistor, 0.000 to $1,111,111.111 \Omega$;

(3) a $0.01 \mathrm{ppm}$ resolution direct reading ratio set for precise 1 to 1 comparisons;

(4) fixed ratio standards with nominal ratios to $10^{6} / 1$; and

(5) an incremental ratio standard having continuous ratio adjustability from $1 / 1$ to $10^{7} / 1$ with 9-dial resolution.

Calibration of the instrument may be accomplished by measuring one of the fixed resistors, and then making 10/1 [5] and/or 100/1 step-up or step-down (ratio) calibrations of the remaining fixed resistors using the technique reported by Hamon [6] and further analyzed by Page [7] or, each of the fixed resistors may be measured and the ratios between them cal-

${ }^{3} \mathrm{~A}$ "tetrajunction" is an electrically symmetrical 4-terminal junction [4] having essentially zero transresistance. culated. The latter has the disadvantage that the uncertainty of the calculated ratios will include the uncertainty in the values of the individual resistors. The decade resistors are calibrated as a 4-terminal resistance box with direct checks of the $\mathrm{xl}$ and $\mathrm{xl0}$ dial settings against the fixed resistors.

\section{URS Modified to a VERI}

A universal ratio set may be modified to permit its additional use as a versatile ratio instrument. A minor modification of a URS for use as a DRRS has been described by Ramaley and Shafer [8]. Figure 3 is a photograph of a URS which has been altered as shown by the schematic diagram of figure 4 . As modified, it may be used as:

(1) a conventional 6-dial URS;

(2) 4-terminal fixed resistance standards, $0.1,1,10$, 100 , and $1000 \Omega$;

(3) a 2- or 4-terminal 6-dial decade resistor, 0.001 , to $2111.110 \Omega$;

(4) a $1 \mathrm{ppm}$ resolution direct reading ratio set for $1 / 1$ comparisons;

(5) fixed ratio standards with nominal ratios to $10^{4} / 1$; and

(6) an incremental ratio standard having continuous ratio adjustability from $1 / 1$ to $2 \times 10^{4} / 1$ with 6 -dial resolution.

The modifications to the URS consist of the following:

(1) Replacing the $\mathrm{x} 100 \Omega$ switch (top decade) with a switch having very low contact resistance variation (less than $0.001 \Omega$ ).

(2) Adding 12 terminals to the front panel, making a total of 17 as seen in figure 3 .

(3) Mounting a tetrajunction (a 1/2-inch diam copper sphere having four terminals).

(4) Mounting the five additional precision resistors, $0.1,1,10,100$, and $1000 \Omega$.

(5) Wiring the resistors and terminals as shown in figure 4. 


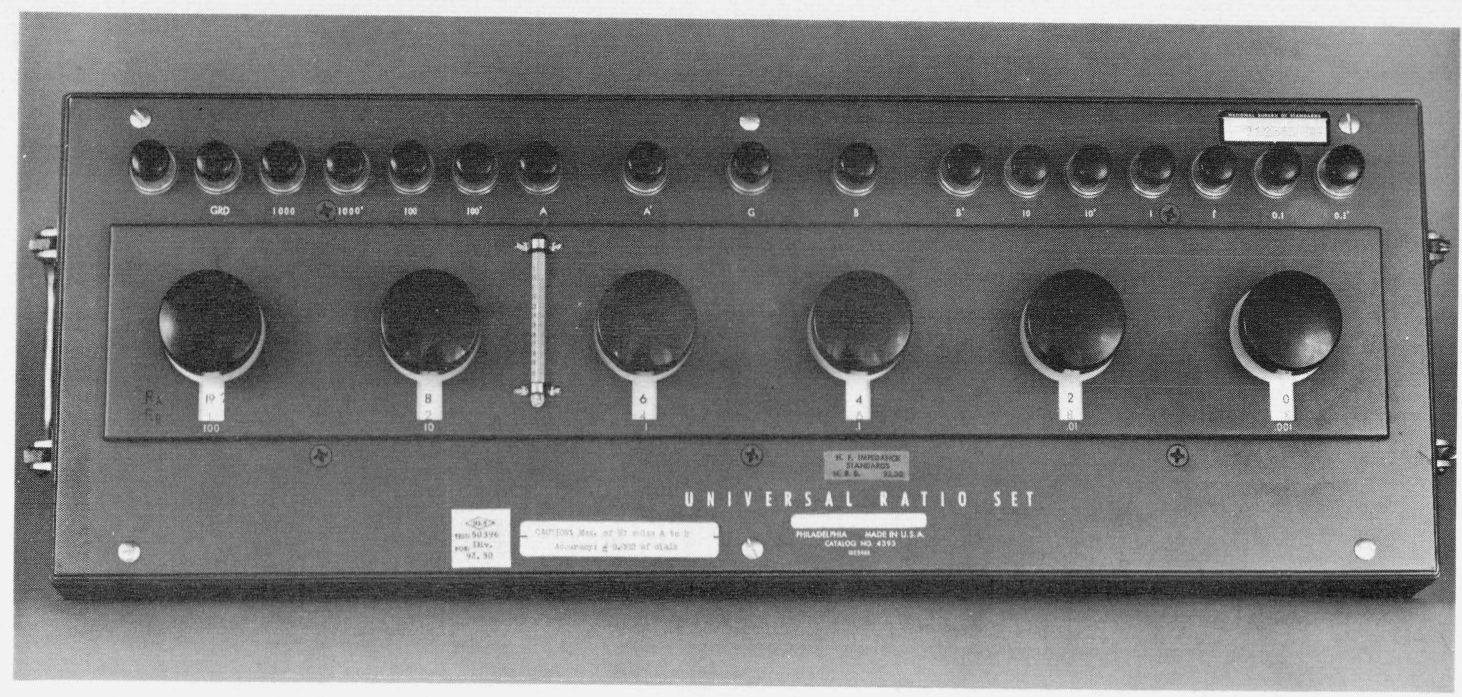

FIGURE 3. A commercial 6-dial URS which has been modified to permit its additional use as a VERI.

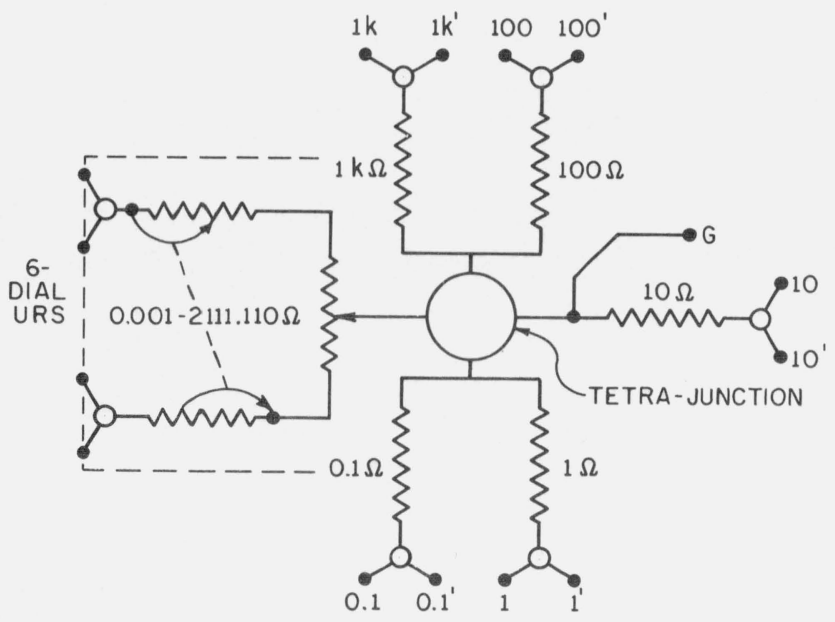

FigURE 4. Schematic diagram of the NBS-modified 6-dial URS to permit its additional use as a VERI.

The spherical tetrajunction, the five added precision resistors, and some of the additional wiring can be seen in the photograph of figure 5 .

\section{A Typical Application of the VERI}

Figure 6 shows a modified 6-dial URS used as a VERI in a setup for calibrating the ratio arms of a Wheatstone bridge. Units $L_{1}$ and $L_{2}$ are lead compensating networks which are needed when resistances of $1000 \Omega$ or less are involved in the ratios. $S_{1}$ and $S_{2}$ are low resistance shorting wires which are used during the adjustment of the lead compensators,
$L_{1}$ and $L_{2}$. Balance is achieved and the setting of the $R_{a}$ dials of the URS is noted when the removal and replacement of the shorting links, $S_{1}$ and $S_{2}$, have no effect on the galvanometer null. The ratio of the Wheatstone bridge ratio arm for the circuit values shown is

$$
M=\frac{R_{a}(1+a)}{0.1(1+b)} \approx \frac{R_{a}}{0.1}(1+a-b)
$$

where $R_{a}$ is the " $R_{a}$ " reading of the URS dials, and $a$ and $b$ are the proportional corrections to $R_{a}$ and $0.1 \Omega$ respectively. (The right-hand expression of the equation introduces less than 1 part in $10^{8}$ uncertainty when $a$ and $b$ are each less than 0.0001.)

Once the ratio arms of the Wheatstone bridge are calibrated, the standard arm, $R_{s}$, may be calibrated by measuring the fixed and decade resistances of the VERI on the "unknown" terminals of the Wheatstone bridge.

\section{Conclusion}

The versatile ratio instrument described permits the precise comparison of ratio from $1 / 1$ to $10^{7} / 1$ with a dial resolution of 1 part in $10^{8}$ or better. By comparison, a standard 6-dial universal ratio set cannot measure a ratio of $10^{7} / 1$, and will give only 3 -digit resolution on a ratio of $10^{4} / 1$. A VERI modified URS, however, will give 7-digit resolution on a ratio of $10^{4} / 1$ and not less than 6-digit resolution on any ratio from $1 / 1$ to $2 \times 10^{4} / 1$. The effective resolution attainable in high ratio measurements may be limited by the maximum permissible generator (battery) voltage and detector (galvanometer) sensitivity. 


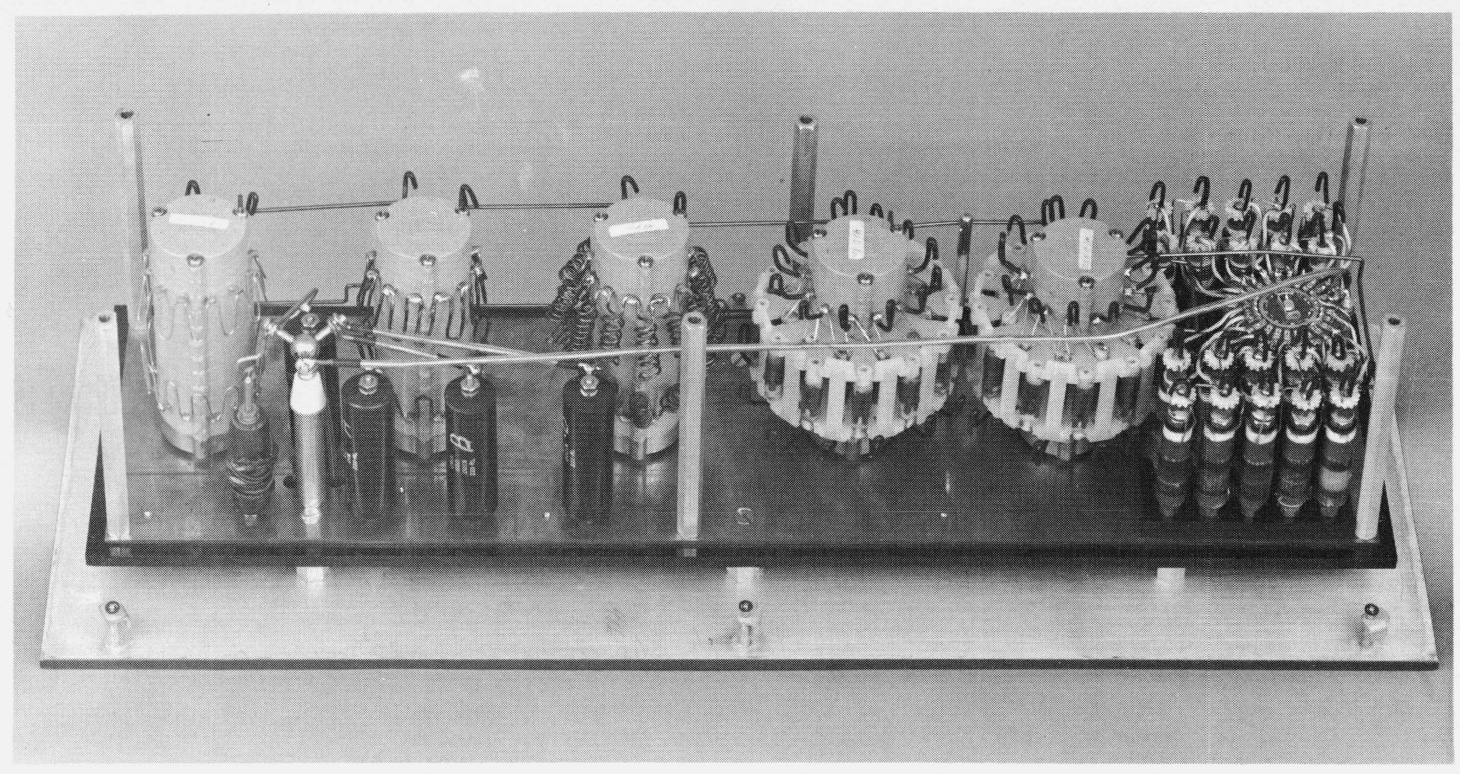

FIGURE 5. Internal view of the commerical 6-dial URS modified by NBS to permit its additional use as a VERI.

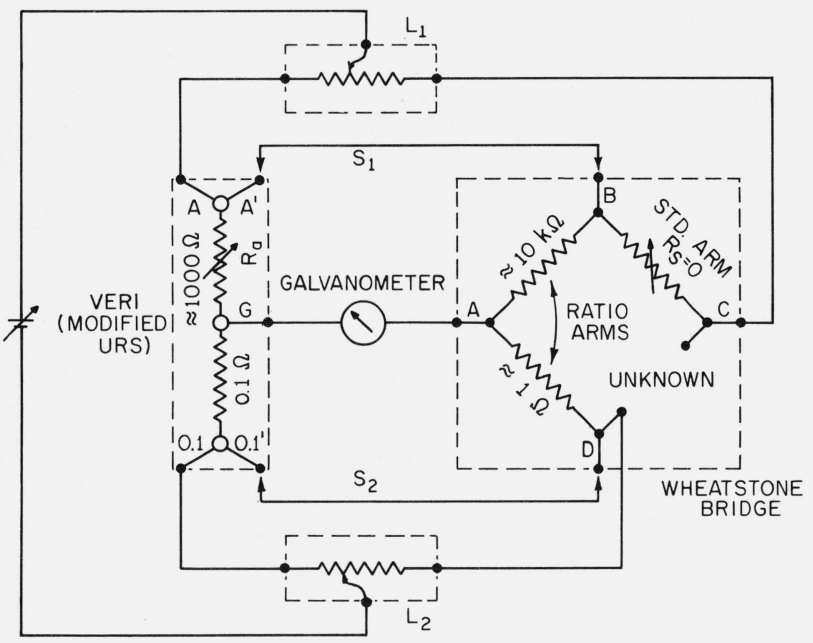

FIGURE 6. Circuit used to calibrate the ratio arms of a Wheatstone bridge with the VERI (modified URS).

\section{References}

[1] Brooks, P. P. B., Calibration procedures for direct current resistance apparatus, NBS Mono. 39 (Mar. 1962).

[2] Thomas, J. L., Precision resistors and their measurements, NBS Circ. 470 (Oct. 1948).

[3] Wenner, F., and E. Weibel, The testing of potentiometers, Scientific Paper 223, Bull. BS 1 1, No. 1, 1-39 (1914).

[4] Hess, A. E., Nano-ohm junctions for accurate ratio instruments, NBS Report No. 9158 (Jan. 1966).

[5] Wenner, F.. Methods, apparatus and procedures for the comparison of precision standard resistors, J. Res. NBS 25, 229293 (Aug. 1940).

[6] Hamon, B. V., A 1-100 $\Omega$ Build-up Resistor for the Calibration of Standard Resistors, J. Sci. Instr. 36, 450-453 (Dec. 1954).

[7] Page, C. H., Errors in the series-parallel build-up of four-terminal resistors, J. Res. NBS 69C (Engr. and Inst.), No. 1, 3, 181-189 (July-Sept. 1965).

[8] Ramaley, D., and J. F. Shafer, Universal ratio sets made direct reading sets, NBS Tech. News Bull. 47, 9, 162 (Sept. 1963).

NOTE: A reprint of references [2] and [5] is also in Precision Measurement and Calibration-Electricity and Electronics, NBS Handbook 77, 1 (1961).

(Paper 70C3-228) 